\title{
Inquérito Nacional sobre as Ligas Acadêmicas de Medicina Intensiva*
}

\section{Survey on Brazilian Critical Care Medicine Undergraduate Study Groups}

\author{
Flávia Branco Cerqueira Serra Neves ${ }^{1}$, Patrícia Sena Vieira ${ }^{1}$, Elaine Andrade Cravo ${ }^{1}$, Maria Dias ${ }^{1}$, \\ Almir Bitencourt'2, Hélio Penna Guimarães ${ }^{3,4,5}$, Gilson Soares Feitosa-Filho ${ }^{4,6}$, José Maria da Costa Orlando ${ }^{4,7}$
}

\section{RESUMO}

JUSTIFICATIVA E OBJETIVOS: A Associação de Medicina Intensiva Brasileira (AMIB) tem estimulado a criação de ligas acadêmicas de Medicina Intensiva (MI), considerando-as instrumento útil no preenchimento de lacunas na formação profissional. O objetivo deste trabalho foi avaliar as atividades desenvolvidas pelas ligas acadêmicas de MI de todo o Brasil.

MÉTODO: Foi realizada análise das informações obtidas pela resposta a um questionário padrão. O contato foi feito por e-mail ou telefone com os representantes das Ligas constantes de lista fornecida pelo Comitê das Ligas Acadêmicas (LIGAMI-AMIB) em setembro

1. Membro da Liga Acadêmica de Medicina Intensiva da Bahia - LAMIB.

2. Ex-Membro da Liga Acadêmica de Medicina Intensiva da Bahia - LAMIB.

3. Ex-Presidente do Comitê da Ligas de Medicina Intensiva-LIGAMIAMIB na gestão de 2004-2005 e 2006 -2007.

4. Título de Especialista em Medicina Intensiva pela AMIB-AMB

5. Médico Diarista da UTI da Disciplina de Clínica Médica da Universidade Federal de São Paulo-UNIFESP-EPM. Coordenador do Centro de Centro de Treinamento em Medicina de Urgência da Sociedade Brasileira de Clínica Médica-SBCM.

6. Membro do Conselho Consultivo da Liga Acadêmica de Medicina Intensiva da Bahia - LAMIB. Membro do Corpo Docente das Residências de Clínica Médica e de Cardiologia do Hospital Santa Izabel da Santa Casa de Misericórdia da Bahia.

7. Ex-Presidente da Associação de Medicina Intensiva Brasileira (AMIB) de 2003-2005 e 2006-2007.

${ }^{*}$ Recebido da Liga Acadêmica de Medicina Intensiva da Bahia - LAMIB, Salvador, BA

Apresentado em 05 de janeiro de 2008

Aceito para publicação em 19 de fevereiro de 2008

Endereço para correspondência:

Flávia Branco Cerqueira Serra Neves

Av. Orlando Gomes, 382, Cond. Village Piatã, Casa B-18, Piatã.

41650-010 - Salvador, BA

Fone: (71) 3367-4427 / 8728-2328

E-mail: flavinhaneves@superig.com.br

(C)Associação de Medicina Intensiva Brasileira, 2008 de 2007. Na época existiam 33 ligas associadas ao LIGAMI, das quais quatro não mais estão ativas, 17 responderam ao questionário enviado. As 12 restantes não responderam ao questionário ou não foi obtido contato.

RESULTADOS: A maioria das ligas foi fundada a partir de 2005, coincidindo com a criação do Comitê LIGAMIAMIB, e são vinculadas a uma ou mais faculdades de Medicina. Dentre as atividades realizadas destaca-se a realização de aulas teóricas (100\%), geralmente ministradas pelos professores orientadores ou médicos convidados $(69 \%)$. Outras atividades incluem práticas em unidade de terapia intensiva (UTI) (88\%), organização de eventos (77\%) e atividades de pesquisa (65\%). A maioria das ligas (65\%), já realizou algum evento científico (cursos, simpósios e jornadas), no entanto, apenas três referiram já ter realizado o Curso de Introdução à Medicina Intensiva da AMIB.

CONCLUSÕES: O número crescente de ligas de MI no Brasil demonstra o interesse dos estudantes por esta especialidade e aponta para a necessidade de maior integração no intuito de trocar experiências, cooperar na realização de atividades de pesquisa e participar de eventos nacionais e internacionais.

Unitermos: Educação Médica, Estudantes de Medicina, Medicina Intensiva, UTI

\section{SUMMARY}

BACKGROUND AND OBJECTIVES: The Brazilian Intensive Care Medicine Association (AMIB) has been stimulating the creation of undergraduate associations/ study groups on Critical Care Medicine (CCM), considering them a useful instrument to fill gaps in professional formation. The aim of this study was to evaluate the activities developed by CCM undergraduate study groups in Brazil.

METHODS: The analyzed information was obtained on answers to a standard questionnaire. Contact by e- 
mail and/or telephone was made with representatives of all study groups registered on AMIB Committee of Undergraduate Study Groups on CCM (LIGAMI-AMIB) in September of 2007. In that period, there were 33 groups associated to LIGAMI, which 4 doesn't exist anymore, 17 answered to the sent questionnaire and the remaining 12 didn't answer the questionnaire or it was not obtained contact.

RESULTS: Most study groups were founded after 2005, coinciding with the LIGAMI-AMIB Committee creation, and they are linked to one or more medical schools. Among the group's activities, the most frequent was theoretical classes (100\%), usually supplied by teachers or invited physicians (69\%). Other activities include practices on Intensive Care Units (88\%), organization of scientific events $(77 \%)$ and research projects (65\%). Most study groups (65\%) had already organized some scientific event such as courses and symposia; however, only three had already accomplished the AMIB CCM Introductory Course.

CONCLUSIONS: The growing number of CCM undergraduate study groups in Brazil demonstrates students' interest for this specialty. Besides, there is a necessity of larger integration between existing groups to change experiences, cooperate in the accomplishment of research projects and participation on national and international events.

Key Words: Critical Care, ICU, Medical Education, Medical Students.

\section{INTRODUÇÃO}

A Medicina Intensiva (MI) é uma especialidade médica relativamente nova, ainda pouco inserida nos cursos de graduação em Medicina1. O aumento do número de pacientes de alta gravidade em unidades de terapia intensiva (UTI), atribuído ao aperfeiçoamento tecnológico, à maior prevalência de doenças crônicas e à crescente incidência de sepse, torna fundamental, portanto, o maior conhecimento da especialidade durante a graduação. $\mathrm{A}$ inclusão da $\mathrm{MI}$ nos currículos da graduação médica vem sendo discutida nas últimas décadas, principalmente pela possibilidade de apresentar ao graduando conhecimentos relacionados às habilidades de reconhecer o paciente grave, iniciar precocemente uma intervenção terapêutica, executar procedimentos e discutir aspectos éticos ${ }^{1-3}$.

A criação das ligas acadêmicas foi uma alternativa encontrada pelos próprios estudantes para suprir a deficiência do ensino de Ml durante a graduação. As ligas são criadas e organizadas por acadêmicos e orientadas por professores e profissionais que apresentem interesses em comum. Elas são constituídas por uma diretoria administrativa e membros efetivos; o número de participantes pode ser variável e sua duração é ilimitada. Todos os integrantes das ligas estão submetidos às normas ditadas pelo estatuto, que deve conter os objetivos, as finalidades, o código disciplinar e as obrigações dos diretores e membros ${ }^{4}$. A difusão da MI na graduação, as oportunidades de exercer atividades práticas e o incentivo à pesquisa clínica, são o cerne da motivação à participação intensa dos alunos nas ligas.

A Associação de Medicina Intensiva Brasileira (AMIB) tem estimulado a criação de ligas acadêmicas de $\mathrm{Ml}$ através do Comitê das Ligas de Medicina Intensiva (LIGAMI-AMIB), pois as considera instrumento útil no preenchimento das lacunas na formação do futuro profissional. O Comitê LIGAMI-AMIB tem como principal finalidade congregar os alunos em seus vários níveis de conhecimento, permitindo maior interesse e aproximação com a MI. Para tanto, o Comitê elaborou um guia que pode ser utilizado para orientar a criação e estruturação de novas ligas acadêmicas de MI nas Instituições de ensino médico em todo país ${ }^{4}$.

O objetivo deste estudo foi avaliar as atividades desenvolvidas pelas ligas acadêmicas de $\mathrm{Ml}$ de todo o Brasil.

\section{MÉTODO}

Foi realizado um estudo descritivo, de corte transversal, no qual foram avaliadas as ligas acadêmicas de MI do Brasil. Solicitaram ao Comitê LIGAMI-AMIB, em setembro de 2007, uma lista com todas as ligas cadastradas e foi feito contato por e-mail e/ou telefone com os representantes de cada liga.

Para todas as ligas contatadas foi enviado por e-mail um questionário sobre suas características e funcionamento, sendo ainda solicitado que fosse enviado seu estatuto. $\mathrm{O}$ questionário foi elaborado considerando os principais tópicos de atividades a serem desempenhadas pelas LIGAMI, de acordo com modelo de estatuto para as Ligas Acadêmicas de MI do Cômite LIGAMI$\mathrm{AMIB}^{4}$. A participação no estudo foi voluntária.

A análise dos dados foi feita através do software Statistical Package for Social Science (SPSS) na versão 9.0. Foram utilizados os parâmetros da estatística descritiva adotando-se as medidas usuais de tendência central e de dispersão, e cálculos de freqüências simples e relativas. 


\section{RESULTADOS}

No período de avaliação deste estudo existiam 33 ligas afiliadas ao Comitê LIGAMI-AMIB, das quais quatro não mais estão ativas no momento e 17 responderam ao questionário enviado. As 12 restantes não responderam ao questionário ou não foi obtido contato efetivo com sucesso. O número de ligas afiliadas ao LIGAMIAMIB em dezembro de 2007 passou a 41.

A maioria das 17 ligas que responderam ao questionário foi fundada a partir de 2005, coincidindo com a criação do Comitê LIGAMI-AMIB, e são vinculadas à pelo menos uma faculdade de Medicina, concentrando-se principalmente na região Sudeste. O número médio de integrantes foi de 23 membros efetivos, sendo que sete ligas aceitam estudantes de outras faculdades. A maioria das ligas só aceita estudantes a partir do $3^{\circ}$ ano do curso e exige tempo mínimo de permanência de um ano, sem tempo máximo predeterminado. As principais características das ligas incluídas estão detalhadas na tabela 1.

O principal critério utilizado para seleção de novos membros é de testes teóricos escritos, utilizados por $88 \%$ das ligas avaliadas. Outros critérios utilizados foram: entrevista (35\%), análise de currículo e histórico escolar (12\%), prova oral (6\%), curso introdutório ou aula inaugural (6\%). O número médio de novos integrantes por ano é 16, variando de 3 a 30 , e três ligas referiram que este número é variável.

Dentre as principais atividades realizadas destaca-se a realização de aulas teóricas (100\%), geralmente mi- nistradas pelos professores orientadores ou médicos convidados $(69 \%)$, mas também há aulas pelos próprios alunos (44\%). Outras atividades incluem práticas em UTI, organização de eventos, atividades de pesquisa e de extensão (Figura 1). As principais obrigações dos integrantes das ligas estão descritas na figura 2. A periodicidade das reuniões geralmente é semanal ou quinzenal (Figura 3). As ligas possuem em média quatro médicos orientadores, que são intensivistas e professores da faculdade. Os médicos orientadores ministram aulas, orientam as atividades práticas e auxiliam na organização de eventos, projetos de pesquisa e extensão. Apenas duas ligas relataram participação de residentes de $\mathrm{Ml}$ em suas atividades.

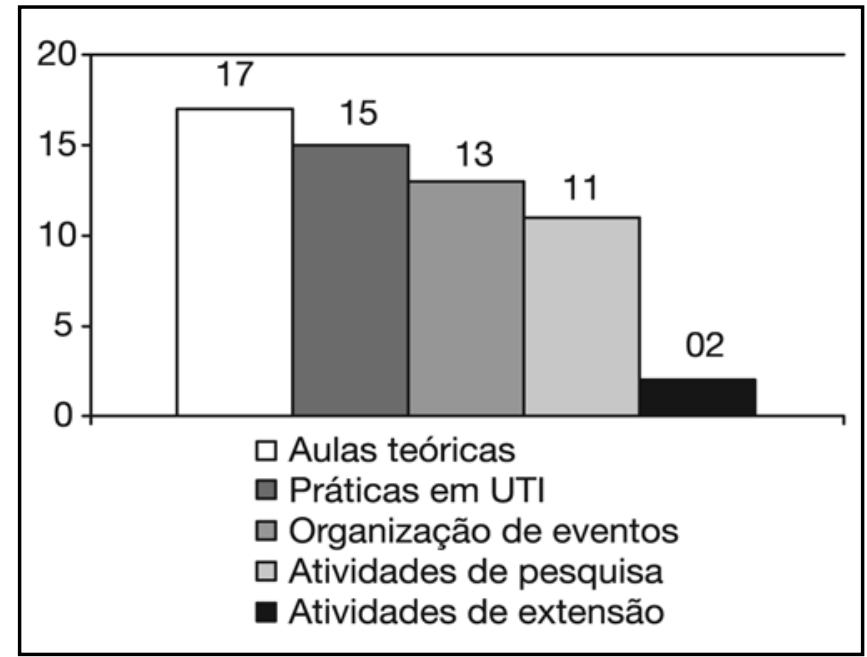

Figura 1 - Atividades Promovidas pelas Ligas Acadêmicas de Medicina Intensiva $(n=17)$.

Tabela 1 - Características das Ligas Acadêmicas de Medicina Intensiva que Responderam ao Questionário

\begin{tabular}{|c|c|c|c|c|c|c|}
\hline Ligas & Estados & Ano de Fundação & Número de Alunos & $\begin{array}{l}\text { Número de Orien- } \\
\text { tadores }\end{array}$ & Restrição & $\begin{array}{l}\text { Tempo Mínimo de } \\
\text { Permanência }\end{array}$ \\
\hline$\overline{1}$ & $\mathrm{SP}$ - Interior & 2001 & 19 & 3 & - & - \\
\hline 2 & SP - Capital & 2003 & 23 & 17 & $5^{\circ} \mathrm{sem}$ & - \\
\hline 3 & SP - Interior & 2003 & 26 & 2 & $2^{\circ} \mathrm{sem}$ & 1 ano \\
\hline 4 & RS & 2004 & 30 & 1 & $5^{\circ} \mathrm{sem}$ & - \\
\hline 5 & $\mathrm{SP}$ - Interior & 2005 & 32 & 4 & $5^{\circ}$ sem. & 1 ano \\
\hline 6 & $\mathrm{BA}$ & 2005 & 16 & 4 & $6^{\circ} \mathrm{sem}$. & 1 ano \\
\hline 7 & $\mathrm{SP}$ - Interior & 2005 & 56 & 6 & $7^{\circ} \mathrm{sem}$ & 1 ano \\
\hline 8 & ES & 2005 & 30 & 1 & $6^{\circ}$ sem. & 1 ano \\
\hline 9 & SP - Interior & 2005 & 20 & 3 & $5^{\circ} \mathrm{sem}$. & 1 ano \\
\hline 10 & RJ - Capital & 2005 & 26 & 2 & $7^{\circ} \mathrm{sem}$ & 1 ano \\
\hline 11 & CE & 2006 & 6 & 2 & $4^{\circ}$ sem. & - \\
\hline 12 & SP - Interior & 2006 & 20 & 4 & - & - \\
\hline 13 & MG & 2006 & 14 & 8 & $7^{\circ}$ sem. & 1 ano \\
\hline 14 & $\mathrm{RO}$ & 2006 & 20 & 7 & $5^{\circ}$ sem. & 2 anos \\
\hline 15 & MS & 2006 & 19 & 1 & $5^{\circ}$ sem. & 1 ano \\
\hline 16 & RJ - Interior & 2007 & 18 & 3 & $7^{\circ}$ sem & 2 anos \\
\hline 17 & RJ - Interior & 2007 & 17 & 4 & $5^{\circ} \mathrm{sem}$. & 1 ano \\
\hline
\end{tabular}




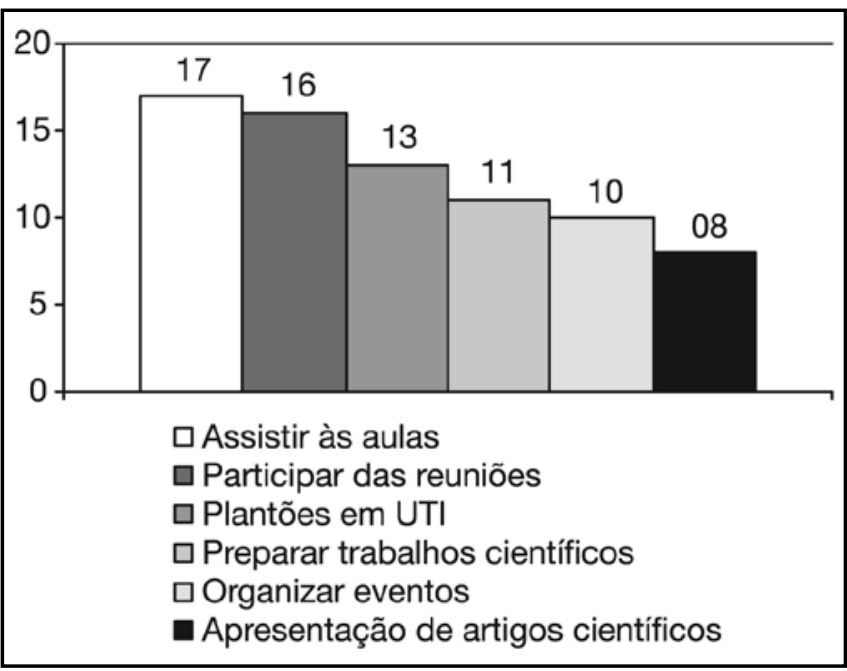

Figura 2 - Obrigações dos Integrantes das Ligas Acadêmicas de Medicina Intensiva $(n=17)$.

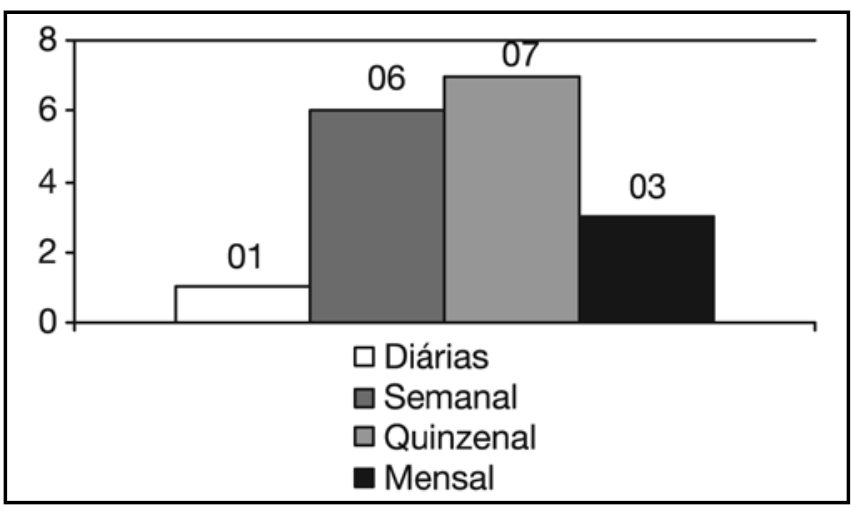

Figura 3 - Periodicidade das Reuniões das Ligas Acadêmicas de Medicina Intensiva $(n=17)$.

A diretoria é renovada anualmente em treze ligas. A maioria das ligas $(65 \%, n=11)$ já realizou algum tipo de evento científico, incluindo cursos, simpósios e jornadas. No entanto, apenas três relataram já ter realizado o Curso de Introdução à Medicina Intensiva da AMIB, a despeito de seus membros terem realizado o curso em eventos de outras regionais ou do próprio comitê LIGAMI.

\section{DISCUSSÃO}

As ligas acadêmicas vêm se tornando importantes elementos na construção do saber do estudante de medicina. Poucos estudos, no entanto, tem avaliado as atividades realizadas pelas ligas e sua importância para a educação médica ${ }^{5}$. No presente estudo verificou-se que desde 2005, houve a criação de maior número de ligas na área. Este incremento parece ser reflexo de diversos fatores, a exemplo do atual crescimento dessa especialidade médica, a conscientização dos profissionais médicos da importância de sua presença na graduação e sua inserção no projeto de reforma da educação médica no Brasil ${ }^{6}$. Outros fatores que podem ter contribuído é o estímulo da AMIB com a criação do Comitê LIGAMI-AMIB, também ocorrido no ano de 2005, bem como a disseminação do conhecimento cada vez maior sobre a própria entidade "liga acadêmica" pelo meio estudantil.

As ligas são entidades que participam de forma efetiva na educação médica, agregando valor à formação do estudante nas áreas de ensino, pesquisa e extensão. Em relação ao ensino, assim como é feito pela maioria das ligas avaliadas, são fundamentais a realização de aulas teóricas, sessões clínicas e eventos científicos em geral com o objetivo de difundir os principais temas relacionados à $\mathrm{Ml}$ entre os estudantes. Estudos prévios já demonstraram que a maioria dos estudantes apresenta grande interesse por estes temas ${ }^{7,8}$.

Uma atividade a ser destacada neste contexto é o Curso de Introdução à Medicina Intensiva, um curso da AMIB voltado para os estudantes de Medicina. Neste curso, são abordados aspectos teórico-práticos básicos comuns à $\mathrm{Ml}$ como intubação traqueal, reanimação cardiopulmonar, acessos venosos, acessos arteriais, monitoração hemodinâmica invasiva, ventilação mecânica básica e terapia nutricional ${ }^{4}$. A principal finalidade é demonstrar, através da prática simulada em manequins, a rotina desses procedimentos, permitindo ao aluno a execução e familiarização com as atividades do atendimento médico de uma UTI. Este curso tem sido bem avaliado pelos estudantes nos locais em que foram realizados ${ }^{9}$. No entanto, foi observada pouca freqüência na sua realização, tendo sido promovido por somente três das ligas avaliadas. Tal situação pode ser decorrente das dificuldades existentes na organização deste curso, principalmente referentes aos gastos e apoio necessários, já que se trata de um evento que implica em aluguel de manequins e materiais para as estações práticas, além de contato com profissionais habilitados para realizá-las.

Observou-se a presença de atividades de pesquisa na maioria das ligas estudadas. Acredita-se que ao experimentar áreas de pesquisa, os estudantes tornam-se potencialmente melhores profissionais pela ampliação de sua visão crítica e de seu poder de reflexão ${ }^{10}$. A formação científica integrada à formação médica é de tamanha importância que há vários anos, países como os EUA, Canadá e Inglaterra têm não só ministrado, como também avaliado, o resultado da introdução 
de programas de iniciação científica durante o curso médico, no desempenho posterior dos profissionais, mesmo que estes não continuem ligados a grupos de pesquisa. Uma das conclusões obtidas foi que pessoas que participaram de bons grupos de pesquisa, mesmo que não continuem com esta atividade, ainda assim tendem a se tornar líderes locais ou regionais. Isto por terem aprendido a se expressar baseados em informações sólidas; por terem adquirido capacidade de análise; e porque seu conhecimento de pesquisa os capacitou a avaliar criticamente artigos científicos ${ }^{10,11}$. As atividades práticas são também desenvolvidas na maioria das ligas. Estas atividades contribuem para a complementação da formação dos acadêmicos nesta especialidade, devendo ser supervisionada e orientada. O acompanhamento da rotina de uma UTI permite a aprendizagem e o aprimoramento de temas em MI, como também proporciona o desenvolvimento do raciocínio clínico rápido e a capacidade de ter iniciativa. Frankel e col. ${ }^{12}$ estudaram os métodos de ensino de escolas dos EUA e sugeriu a realização de um rodízio em UTI na graduação para facilitar a aquisição de habilidade em conceitos e procedimentos de Ml que proporcionem maior segurança aos futuros médicos no atendimento a pacientes graves. Em contrapartida, os manuais de orientação americanos mostram que apenas uma minoria das escolas médicas exige a participação formal de estudantes em estágio em UTI ${ }^{13}$. O número de integrantes, periodicidade das reuniões e tempo mínimo de permanência variam amplamente e estão relacionados à dinâmica das atividades de cada liga. Os critérios utilizados para seleção de novos membros também variam entre as ligas. Apesar de a maioria realizar uma prova escrita, a associação desta forma de avaliação com outros critérios como participação em curso introdutório, entrevista, análise de currículo ou histórico pode ajudar a selecionar os estudantes que possuam maior interesse pelas atividades da liga e pela especialidade de MI.

Acredita-se também, conforme afirmado por Kara-José e col. ${ }^{5}$, que a diretoria da liga deve ser renovada a cada ano, o que é importante para a divisão de responsabilidades entre os membros, além de contribuir para constante renovação e continuidade da liga após a saída dos membros mais antigos.

$\mathrm{Na}$ maioria das ligas são aceitos acadêmicos apenas a partir do $3^{\circ}$ ano do curso. Este pré-requisito, provavelmente, está relacionado com o próprio currículo da maioria das escolas médicas, correspondendo ao início do ciclo clínico, época em que os estudantes são inseridos em estágios ambulatoriais ou hospitalares. No entanto, deve-se destacar a importância de discutir os temas relacionados à $\mathrm{Ml}$ desde o início do curso. Em 1994, o Council on Graduate Medical Education mencionou a necessidade de reformular o currículo generalista da graduação, incluindo temas de $\mathrm{Ml}$ durante os primeiros anos do curso, dentro de matérias básicas como patologia, farmacologia e fisiologia ${ }^{14}$. A Seção de Cirurgia da Society of Critical Care Medicine (SCCM) também recomenda, através de diretriz publicada em 2000, que a graduação deve incluir conhecimentos sobre a fisiologia básica de doenças graves e o entendimento de conceitos como disfunção de órgãos e resposta inflamatória ao trauma e infecção ${ }^{15}$. Além disso, vale ressaltar que as atividades das ligas podem ser diferenciadas a depender do semestre em curso de cada membro.

\section{CONCLUSÃO}

O número crescente de ligas acadêmicas de MI no Brasil demonstra o interesse dos estudantes por esta especialidade e aponta para a necessidade de uma maior integração no intuito de trocar experiências, cooperar na realização de atividades de pesquisa e participar de eventos nacionais e internacionais. A AMIB, através do Comitê LIGAMI-AMIB, parece ter desempenhado importante papel no incentivo à formação e manutenção das ligas. Espera-se que com o aperfeiçoamento e sedimentação das ligas, a AMIB consiga fortalecer o interesse pela especialidade e incentivar a formação de novos intensivistas.

\section{AGRADECIMENTOS}

Agradecemos a todas as ligas acadêmicas que responderam ao questionário e colaboraram com o estudo; a Luiz Fernando dos Reis Falcão, membro do Comitê das Ligas Acadêmicas de Medicina Intensiva (LIGAMIAMIB), pela disponibilidade e apoio para a execução deste trabalho; e a todos os colegas, integrantes da Liga Acadêmica de Medicina Intensiva da Bahia (LAMIB), pelo incentivo e sugestões.

\section{REFERÊNCIAS}

01. Moraes APP, Araújo GF, Castro CA - Terapia intensiva na graduação médica: os porquês. Rev Bras Ter Intensiva, 2004;1:45-48.

02. Goldwaser R - Como está a Medicina intensiva na graduação médica? Atualidades AMIB, 2003;31:2.

03. Goldwaser R - Educação em Medicina Intensiva. Atualidades AMIB, 2003;27:1. 
04. Guimarães HP, Ferreira ALA, Falcão LFR - Introdução à Medicina Intensiva, $2^{\mathrm{a}} \mathrm{Ed}$, São Paulo: Comitê Especial das Ligas de Medicina Intensiva, 2006.

05. Kara-Jose AC, Passos LB, Kara-José FC et al - Ensino extracurricular em Oftalmologia: grupos de estudos / ligas de alunos de graduação. Rev Bras Educ Med, 2007;31:166-172.

06. Brasil. Mistério da Educação. Conselho Nacional de Educação. Minuta de Resolução, de 3 de outubro de 2001. Institui as Diretrizes Curriculares Nacionais dos Cursos de graduação em Medicina. Boletim ABEM, 2001;29:9-11.

07. Almeida AM, Albuquerque LC, Bitencourt AGV et al - Medicina Intensiva na graduação médica: perspectiva do estudante. Rev Bras Ter Intensiva, 2007;19:456-462.

08. Moraes APP - Terapia Intensiva na graduação médica: nova abordagem, antigas reflexões São Luís: Universidade Federal do Maranhão; 2003.

09. Guimarães HP, Orlando JMC, Ferreira ALA et al - Introdução à Medicina Intensiva para graduação médica: resultados iniciais do Curso LIGAMI-
AMIB. Rev Bras Ter Intensiva, 2006;16:(Suppl):P-127.

10. Fava-de-Moraes F, Fava M - A iniciação científica: muitas vantagens e poucos riscos. São Paulo Perspec, 2000;14:73-77.

11. Does research make for better doctors? Lancet, 1993;342:1063-1064.

12. Frankel HL, Rogers PL, Gandhi RR et al - What is taught, what is tested: findings and competency-based recommendations of the Undergraduate Medical Education Committee of the Society of Critical Care Medicine. Crit Care Med, 2004;32:1949-1956.

13. Shen J, Joynt GM, Critchley LA et al - Survey of current status of intensive care teaching in English-speaking medical schools. Crit Care Med, 2003;31:293-298

14. Spevetz A, Brilli RJ, Warren $\mathrm{J}$ et al - Guidelines for resident physician training in critical care medicine. Crit Care Med, 1995;23:1920-1923.

15. Ivy $\mathrm{M}$, Angood $\mathrm{P}$, Kirton $\mathrm{O}$ et al - Critical care medicine education of surgeons: recommendations from the Surgical Section of the Society of Critical Care Medicine. Crit Care Med, 2000;28:879-880. 